\title{
Comparative Study of IV Esmolol, IV Diltiazem, and IV Lignocaine Hydrochloride in Attenuating Pressure Response to Laryngoscopy and Intubation
}

\author{
Sathya Narayanan Karunanithi ${ }^{1}$, Geeta Bhandari², Kedar Singh Shahi ${ }^{3}$, Aditya Kumar Chauhan ${ }^{4}$, Pooja Gautam ${ }^{5}$ \\ 1Department of Anaesthesiology, Critical Care, Pain and Palliative Medicine Government Medical College, \\ Haldwani, Uttarakhand, India. ${ }^{2}$ Department of Anaesthesiology, Critical Care, Pain and Palliative Medicine \\ Government Medical College, Haldwani, Uttarakhand, India. ${ }^{3}$ Department of Surgery, Government Medical \\ College, Haldwani, Uttarakhand, India. ${ }^{4}$ Department of Anaesthesiology, Critical Care, Pain and Palliative \\ Medicine Government Medical College, Haldwani, Uttarakhand, India. ${ }^{5}$ Department of Anaesthesiology, Critical \\ Care, Pain and Palliative Medicine Government Medical College, Haldwani, Uttarakhand, India.
}

\section{ABSTRACT}

\section{BACKGROUND}

Not many studies have compared more than two drugs in attenuating pressor responses to laryngoscopy and intubation. This study compares four groups of considerable size. The present study compared intravenous esmolol, diltiazem, and lignocaine, for their efficacy to abate pressure response to laryngoscopy and intubation.

\section{METHODS}

This is a prospective, randomized, double-blinded, controlled clinical study conducted among 220 patients of ASA grade I/II (age 18-60 years), undergoing elective surgical procedure requiring general anaesthesia with endotracheal intubation over a period of 15 months at a tertiary hospital setup. Study subjects were categorised as Groups D, E, L, and $\mathrm{N}$ that received diltiazem $(0.2 \mathrm{mg} / \mathrm{Kg} \mathrm{IV})$, esmolol (2 mg/Kg IV), lignocaine (1.5 mg/Kg IV), and normal saline, respectively; each group had 55 patients. Haemodynamic parameters were recorded at baseline, after drug administration, immediately after intubation, and at 1-, 3-, and 5-minutes after intubation. Data entry and analysis were performed using MS Excel and SPSSPC-17 version, respectively. One-way ANOVA and the chi-square test were used to evaluate the difference. $\mathrm{P}<0.05$ was considered significant.

\section{RESULTS}

A maximum increase in haemodynamic parameters occurred immediately after intubation. The increase in heart rate and rate pressure product were significantly lower in Group E $(+2.93 \%$ \& $+15.31 \%)$, whereas the increase in blood pressure was lower in Group D (8.51\%).

\section{CONCLUSIONS}

Haemodynamic stability during laryngoscopy and endotracheal intubation is an integral and essential goal of any anaesthetic management plan and was more effectively maintained with esmolol and diltiazem than with lignocaine.

\section{KEY WORDS}

Diltiazem, Esmolol, Lignocaine, Haemodynamic Response, Intubation, Laryngoscopy, Normal Saline, Intravenous

\author{
Corresponding Author: \\ Dr. Geeta Bhandari, \\ Professor and HOD, \\ Department of Anaesthesiology, \\ Critical Care, Pain and Palliative Medicine, \\ Government Medical College, \\ Haldwani-263139, Uttarakhand, India. \\ E-mail:dr.geetabhandari@gmail.com
}

DOI: $10.14260 /$ jemds/2020/447

How to Cite This Article:

Karunanithi SN, Bhandari G, Shahi KS, et al. Comparative study of IV esmolol, IV diltiazem, and IV lignocaine hydrochloride in attenuating pressure response to laryngoscopy and intubation. J. Evolution Med. Dent. Sci. 2020;9(29):2053-2057, DOI: 10.14260/jemds/2020/447

Submission 30-04-2020,

Peer Review 14-06-2020

Acceptance 20-06-2020,

Published 20-07-2020.

Copyright (C) 2020 JEMDS. This is an open access article distributed under Creative Commons Attribution License [Attribution 4.0 International (CC BY 4.0)] 


\section{BACKGROUND}

Endotracheal intubation has become an integral part of airway management since its description in 1921 by Rowbotham and Magill.[1] Laryngeal, tracheal, and bronchial receptors are stimulated by mechanical and chemical irritants during laryngoscopy, intubation, and extubation. ${ }^{[2]}$

Surges in blood pressure and heart rate (HR) due to reflex sympathetic and vagal discharges after laryngotracheal stimulation often increase the plasma norepinephrine concentration. ${ }^{[3]}$ This change is of limited significance in healthy patients but may prove fatal in patients with cardiovascular instability, causing sudden deaths. ${ }^{[4]}$

These cardiovascular responses may have serious consequences, including myocardial ischemia, dysrhythmias, pulmonary oedema, sudden left ventricular failure, cerebrovascular haemorrhage and at times even cardiac arrest. Hence, abating the pressure response to laryngoscopy and intubation has become one of the most researched topics in the field of anaesthesiology. These changes are tolerated quite well by healthy patients, however patients suffering from coronary artery disease, hypertension, valvular heart disease, stroke, intracranial lesions, and penetrating eye injuries are not able to withstand them. In these patients, myocardial reserve is decreased, and tachycardia associated with laryngoscopy and intubation cause myocardial ischemia.[5]

Numerous attempts have been made to modify haemodynamic responses, including the use of premedication; deep anaesthesia; topical anaesthesia; ganglion blockers and beta blockers,[5] antihypertensive agents such as phentolamine,[6] adjuvants like gabapentin[7] and pregabalin,[8] and vasodilators such as magnesium, Sodium nitroprusside and nitroglycerine ${ }^{[9]}$ are effective in modifying the responses but require continuous monitoring.

Various drug regimens and modalities, including opioids, barbiturates, benzodiazepines, beta blockers, calcium channel blockers, and vasodilators, have been commonly used for blunting the stress response to laryngoscopy and intubation. ${ }^{[10-12]}$ However, none of them have proved to be ideal due to their limitations and side effects. Thus, the search for an ideal agent to negate the haemodynamic responses of laryngoscopy and intubation has never ceased.

Esmolol, [methyl 3-\{4-(2-hydroxyl-3)-isopropylamino propoxyl] phenyl $\}$ proprionate $\mathrm{HCl}]$ is a cardioselective water soluble ultrashort acting $\alpha-1$ adrenergic receptor antagonist that can be administered only intravenously.

Esmolol is rapidly hydrolysed by cytoplasmic esterases in red blood cells and hence has a short elimination period of approximately $9 \mathrm{~min}$., a distribution half-life of $2 \mathrm{~min}$ and a peak haemodynamic effect within 6 to $10 \mathrm{~min}$. of administration. Its metabolism is not influenced by renal or hepatic function and less than $1 \%$ excreted in urine as unchanged drug.[13]

Esmolol is a striking option because of its cardioselectivity and ultra-short duration of action, but it can only be administered intravenously.[14]

Calcium channel blockers are also preferred because myocardial depression produced by them is minimized by reduction in afterload so that cardiac output remains unchanged, but they show no effect on increase in heart rate.
The drug should be used with caution in patients with impaired renal or hepatic function.[15-17]

Lidocaine is [2-(Diethylamino)-N-2, 6-Dimethyl phenyl acetamide)] an amide group of local anaesthetic agent. It is metabolized by oxidases and amidases from microsomes of liver and the metabolites are excreted in the urine, hastened when the urine is acidic.[13]

Only a few studies with compact sample sizes comparing the efficacy of intravenous (IV) lidocaine, esmolol, and diltiazem for abolishing the pressure response have been conducted to the best of our knowledge. Hence, the present study with a substantial sample size was planned to compare the aforesaid.

\section{METHODS}

The present study was conducted for 15 months from March 2018 to May 2019 in our hospital after receiving an approval from the institutional ethics committee. This prospective, randomized, double-blind, controlled clinical study comprised 220 normotensive consented male and female patients aged $18-60$ years.

The sample size is calculated by taking mean of the pulse rate after the drug administration from the following studies using WIN PEPI software.

1. The mean of pulse rate in the diltiazem group was taken as 94 after drug administration.[18]

2. The mean of pulse rate in the lignocaine group was taken as 81.76 and that of esmolol as 82.96 after drug administration.[19]

Sample size has been calculated by using power analysis \& alpha error of 0.05 and the power of the study as $80 \%$.

They were scheduled for an elective surgery under general anaesthesia with endotracheal intubation of ASA physical status I and II. Patients with ASA grade III or more; receiving beta blockers or calcium channel blockers; with significant hepatorenal disease, uncontrolled hypertension, diabetes, and significant respiratory or cardiovascular diseases; posted for emergency surgeries; undergoing difficult tracheal intubation; and who are underweight or obese were excluded from the study.

After a meticulous preanaesthetic examination, the patients were randomly divided into Groups D, E, L, and $\mathrm{N}$ of 55 each by using a closed envelope technique.

The name of 4 groups of drugs were equally written in 220 opaque envelopes and sealed. The sealed envelopes were placed in a container and shuffled. After shuffling, the sealed envelopes were numbered from 1 to 220 in sequence. Sealed envelope were opened in sequence corresponding to the number of the patient. Double blinding was done to avoid any bias.

Groups D, E, and L received an injection of diltiazem $(0.2$ $\mathrm{mg} / \mathrm{Kg}$ ), esmolol (2 mg/Kg), and lignocaine $(1.5 \mathrm{mg} / \mathrm{Kg})$, respectively, whereas Group C received $3 \mathrm{~mL}$ normal saline.

All the groups received the aforementioned as a bolus intravenously 2 min before laryngoscopy and intubation.

Upon arrival in the operation theatre, all noninvasive monitors were secured to the patients, and their vitals were 
monitored. The appropriate IV fluid was started, along with Ringer's Lactate solution. All the patients were premedicated with glycopyrrolate $(0.2 \mathrm{mg}$, IV) and midazolam $(0.5-1 \mathrm{mg}$, IV) before anaesthesia induction. The HR and systolic and diastolic blood pressures were recorded as the baseline value.

All patients were preoxygenated for 3 min with $100 \%$ oxygen through Bain's circuit. After study drug administration, anaesthesia was induced with IV propofol (1\%, $2.5 \mathrm{mg} / \mathrm{Kg})$, followed by succinylcholine $(1.5 \mathrm{mg} / \mathrm{Kg})$.

Laryngoscopy was performed using a Macintosh laryngoscope, and intubation was performed using an appropriately sized, disposable, high-volume low-pressure cuffed endotracheal tube. Laryngoscopy and intubation were performed within 15-20 s, with a strict and vigil monitoring of haemodynamic parameters-the HR, arterial blood pressure, and electrocardiographic changes which were observed at baseline, after drug administration, immediately after intubation, and at 1, 3, and 5 min after intubation.

These parameters were noted by anaesthesiologist who was blinded about the study drug. Providing any surgical stimulus was avoided during the study period.

Anaesthesia was maintained with a balanced technique of $66 \%$ nitrous oxide, $33 \%$ oxygen, $1 \%$ isoflurane, or $0.5 \%$ halothane, and a nondepolarizing muscle relaxant, vecuronium, one-fourth of the loading dose given at an appropriate time and IPPV. Ventilation adequacy was monitored clinically with ETCO2, and $\mathrm{SpO} 2$ was maintained at $99 \%-100 \%$.

At the end of the surgery, neuromuscular blockade was reversed with injection neostigmine $(0.05 \mathrm{mg} / \mathrm{Kg})$ and injection glycopyrrolate $(0.008-0.01 \mathrm{mg} / \mathrm{Kg})$. Extubation was performed after the return of the protective airway reflex, and the patients were shifted to the recovery room for further observation. The patients were observed for drugrelated adverse effects and anaesthesia-related problems and were attended accordingly.

\section{Statistical Analysis}

The collected data were transformed into variables, coded and entered in Microsoft Excel. Data was analysed and statistically evaluated using SPSS version 17 (Chicago II, USA). Quantitative data were expressed as mean and standard deviation, and differences between the groups were determined using one-way ANOVA or the Kruskal-Wallis $\mathrm{H}$ test, followed by the post hoc test, whereas qualitative data were expressed as percentages. Statistical differences between the proportions were tested using the chi-square test or Fisher's exact test. $\mathrm{p}<0.05$ was considered significant.

\section{RESULTS}

The demographic profiles of all groups were comparable (table 1). From drug administration to the study observation period, the HR was significantly lower in Group E, followed by Group D and Group L. The maximum increase in HR was observed immediately after laryngoscopy (table 2). Following

\begin{tabular}{|cccccc|}
\hline & Group D & Group E & Group L & Group C & P-Value \\
Age (in years) & $36.05 \pm 11.48$ & $33.60 \pm 11.76$ & $37.51 \pm 11.72$ & $34.16 \pm 12.51$ & 0.29 \\
Gender (M/F) & $13 / 42$ & $13 / 42$ & $9 / 46$ & 17.38 & 0.36 \\
Weight (Kgs) & $60.31 \pm 8.57$ & $58.56 \pm 7.89$ & $59.62 \pm 9.27$ & $59.25 \pm 7.08$ & 0.73 \\
ASA (I/II) & $32 / 23$ & $35 / 20$ & $30 / 25$ & $34 / 21$ & 0.77 \\
\hline \multicolumn{5}{r}{ Table 1. Demographic Profile of Study Subjects } \\
\hline
\end{tabular}

\begin{tabular}{|cccccc|}
\hline Heart Rate & Group D & Group E & Group L & Group C & $\begin{array}{c}\text { P- } \\
\text { Value }\end{array}$ \\
$\begin{array}{c}\text { Basal } \\
\text { After drug } \\
\text { administration } \\
\text { Immediately } \\
\text { after }\end{array}$ & $78.11 \pm 6.82$ & $79.09 \pm 5.92$ & $77.02 \pm 6.36$ & $79.49 \pm 8.04$ & 0.35 \\
intubation & $93.65 \pm 4.17$ & $81.24 \pm 7.54$ & $96.67 \pm 10.33$ & $102.91 \pm 12.98$ & $<0.001$ \\
After 1 min & $92.58 \pm 5.11$ & $80.73 \pm 7.74$ & $97.51 \pm 9.96$ & $107.60 \pm 11.31$ & $<0.001$ \\
After 3 min & $92.36 \pm 6.28$ & $80.58 \pm 6.87$ & $97.09 \pm 10.09$ & $108.11 \pm 10.88$ & $<0.001$ \\
After 5 min & $82.22 \pm 7.16$ & $80.47 \pm 7.43$ & $90.87 \pm 9.63$ & $97.16 \pm 10.67$ & $<0.001$ \\
\hline Table 2. Changes in Heart Rate in Control and Experimental Groups \\
\hline \multicolumn{7}{|c|}{} \\
\hline
\end{tabular}

\begin{tabular}{|cccccc|}
\hline MAP & Group D & Group E & Group L & Group C & P-Value \\
Basal & $95.29 \pm 8.62$ & $97.69 \pm 4.14$ & $95.31 \pm 4.44$ & $96.58 \pm 4.06$ & 0.26 \\
$\begin{array}{c}\text { After drug } \\
\text { administration }\end{array}$ & $91.38 \pm 9.36$ & $90.05 \pm 6.87$ & $90.15 \pm 6.07$ & $90.78 \pm 5.54$ & 0.34 \\
$\begin{array}{c}\text { Immediately } \\
\text { after intubation }\end{array}$ & $102.53 \pm 9.71$ & $105.40 \pm 4.47$ & $110.56 \pm 3.25$ & $112.62 \pm 3.90$ & $<0.001$ \\
After 1 min & $101.45 \pm 7.80$ & $105.42 \pm 4.09$ & $110.62 \pm 5.04$ & $112.18 \pm 4.39$ & $<0.001$ \\
After 3 min & $99.53 \pm 9.18$ & $101.29 \pm 13.36$ & $104.95 \pm 6.97$ & $106.85 \pm 5.64$ & $<0.01$ \\
After 5 min & $96.04 \pm 11.04$ & $102.13 \pm 5.75$ & $102.67 \pm 6.51$ & $103.16 \pm 4.53$ & $<0.01$ \\
\hline \multicolumn{5}{c}{ Table 3. Changes in Mean Arterial Pressure } \\
in Control and Experimental Groups \\
\hline
\end{tabular}

\begin{tabular}{|cccccc|}
\hline RPP & Group D & Group E & Group L & Group C & $\begin{array}{c}\text { P- } \\
\text { Value }\end{array}$ \\
Basal & $9910.75 \pm$ & $9873.02 \pm$ & $9798.73 \pm$ & $10097.75 \pm$ & 0.42 \\
After drug & 1131.27 & 778.07 & 872.31 & 1096.02 & \\
administration & 1273.62 & 1167.01 & 1022.89 & 2683.88 & 0.04 \\
Immediately & $13624.51 \pm$ & $11339.20 \pm$ & $14000.07 \pm$ & $15602.78 \pm$ & \\
after & 1476.58 & 1311.22 & 1633.86 & 2261.51 & $<0.001$ \\
intubation & $12840.15 \pm$ & $11255.93 \pm$ & $13274.76 \pm$ & $15348.04 \pm$ & \\
After 1 min & 1093.04 & 1417.45 & 1646.90 & 1789.76 & $<0.001$ \\
& $12728.45 \pm$ & $10875.42 \pm$ & $13108.73 \pm$ & $14880.89 \pm$ & $<0.001$ \\
After 3 min & 1479.44 & 1198.55 & 1748.70 & 1761.37 & \\
& $10386.15 \pm$ & $10657.16 \pm$ & $12379.60 \pm$ & $13086.04 \pm$ & $<0.01$ \\
After 5 min & 1420.36 & 1187.97 & 1516.40 & 1673.13 & \\
\hline \multicolumn{7}{c}{ Table 4. Changes in Rate Pressure Product } & \\
\hline & in Control and Experimental Groups & \\
\hline \multicolumn{7}{c}{} \\
\end{tabular}

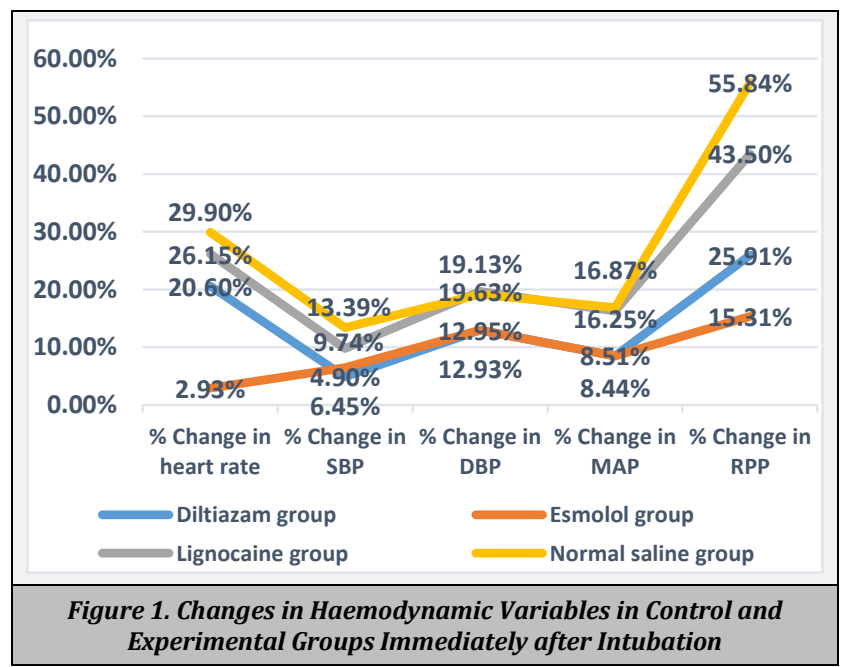

laryngoscopy and intubation, the maximum increase in mean arterial pressure (MAP) was observed in Group N compared with the other groups. In Groups D, E, L, and N, the maximum increase in MAP from the baseline values was observed immediately after intubation. No significant difference in 
MAP was observed between Groups E and D up to 3 min after intubation, except at $1 \mathrm{~min}$ (table 3).

The rate pressure product (RPP) was significantly lower in Group E, followed by Groups D, L, and N. The maximum mean difference was calculated immediately after laryngoscopy and intubation. Statistically significant blunting of laryngoscopic and intubating stress responses, as indicated by the RPP, was reported immediately after intubation, and at 1, 3, and 5 min in Group E, followed by Groups D, L, and N (table 4).

The maximum increase in haemodynamic parameters was observed immediately after intubation. The increase in HR in Groups N, E, D, and L from the baseline to immediately after laryngoscopy and intubation was $+29.9 \%,+2.93 \%$, $+20.6 \%$, and $+26.15 \%$, respectively. The maximum increase in MAP immediately after laryngoscopy and intubation was $16.87 \%$ in Group N, saline, $16.25 \%$ in Group L, $8.44 \%$ in Group E, and $8.51 \%$ in Group D. The maximum increase in RPP immediately after laryngoscopy and intubation was $+55.84 \%$ in Group N, $+15.31 \%$ in Group E, $25.91 \%$ in Group $\mathrm{D}$, and $43.50 \%$ in Group L (figure 1).

No arrhythmia, myocardial ischemia, or hypoxemia was observed in any of the groups, and no serious complications after laryngoscopy and tracheal intubation were reported in any patient.

\section{DISCUSSION}

The haemodynamic response to laryngoscopy and intubation is well established. Various methods have been used to negate these responses. The present clinical study compared the efficacy of three drugs diltiazem, esmolol, and lignocaine to abate pressure response to laryngoscopy and intubation.

Esmolol is a cardio selective beta blocker having an ultrashort duration of action and has been indicated for tachycardia and hypertension during tracheal intubation. [5] For Group E, when compared with their preoperative values (table 2), a non-significant increase $(\mathrm{p}<0.05)$ in the HR was observed only immediately after intubation and at 1 and 3 min after intubation.

At 5 min after intubation, the postoperative values were nearly the same as the preoperative values $(P>0.05)$. Compared with Group N, Group E exhibited significantly less increase immediately after intubation $(p<0.001)$, and at 1 $\min (\mathrm{p}<0.001), 3 \min (\mathrm{p}<0.001)$, and $5 \min (\mathrm{p}<0.001)$ after intubation (table 2). These findings are similar to those of Menkhaus et al.[20] (1985) who found that esmolol administered through continuous infusion abated the HR response at 1, 3, and $4 \mathrm{~min}$ after laryngoscopy and intubation.

Another study by Agrawal et al.[13] reported that compared with their preoperative HR values, the esmololtreated group exhibited a significant increase in the HR only 1 and 2 min after intubation. At $4 \mathrm{~min}$ after intubation, the postoperative HR values were lower than the preoperative values, although the decrease in values was not significant ( $p$ $>0.05$ ).

In the present study, the maximum increase in MAP from the baseline values in Groups D, E, L, and N was observed immediately after intubation.
In Group L, the HR significantly increased from the preoperative value to immediately after intubation, and at 1 $\min (\mathrm{p}<0.001), 3 \mathrm{~min}$, and $5 \mathrm{~min}$ after intubation $(\mathrm{p}<0.01)$. Agrawal et al.[13] found that the lidocaine-treated group also exhibited a significant increase in the HR from the baseline up to $4 \mathrm{~min}$ after intubation; at $5 \mathrm{~min}$ after intubation, no significant difference was observed compared with the baseline.

The percentage changes reported by Singh et al.[21] in MAP from the baseline to $1 \mathrm{~min}$ after intubation were $20.83 \%, 15.89 \%, 10.90 \%$, and $10.20 \%$ in the control, lidocaine, diltiazem, and esmolol groups, respectively. Gupta et al.[22] reported no significant difference in MAP between Groups E and D up to 7 min after intubation. Parvez et al.[23] reported that MAP increased in the control group and persisted for $5 \mathrm{~min}$; however, esmolol more efficiently attenuated the increase in MAP than diltiazem, whereas in the present study, diltiazem more efficiently attenuated the increase than esmolol.

The maximum increase in RPP immediately after laryngoscopy and intubation in Groups N, E, D, and L was $+55.84 \%,+15.31 \%, 25.91 \%$, and $43.50 \%$, respectively. Singh et al.[21] found a marked elevation in RPP in the control group compared with the lidocaine, diltiazem, and esmolol groups after laryngoscopy and intubation.

The differences in results could be due to the use of a higher dose $(2 \mathrm{mg} / \mathrm{Kg}$ ) of esmolol and various induction agents such as thiopentone sodium. Similar to our study, Gupta et al.[22] also reported significantly lower RPPs in the esmolol group than in the diltiazem and lidocaine groups.

Sarkar et al.[24] reported that the increase in RPP was greater with esmolol than with diltiazem, indicating that diltiazem controls the myocardial oxygen demand more efficiently than esmolol. Parvez et al.[23] found a significant difference in RPPs between the esmolol and diltiazem groups at different time intervals. The esmolol group revealed lower values at all-time intervals.

The haemodynamic changes in HR, MAP, and RPP from the baseline value to $1 \mathrm{~min}$ after tracheal intubation in Groups E and D were significantly less compared with those in Group L. Our failure to detect any significant effect of lidocaine compared with esmolol on stress response could be due to the non-inclusion of patients with heart disease in our study, whereas Stoelting et al.[25] included patients with heart disease in their study and reported a favourable response.

Studies have revealed an increase in the incidence of myocardial infarction when the intraoperative HR is more than 110 beats/min. ${ }^{[26]}$

\section{CONCLUSIONS}

Esmolol and diltiazem are comparable in their effect of attenuating sympathetic surge following laryngoscopy and intubation, which is beneficial in patients with ischemic heart disease and hypertension as well as in those undergoing ophthalmic and neurosurgeries. The lack of invasive blood pressure monitoring and no appropriate consensus for the optimal dose and timing of administration of the three drugs are some of the limitations of the present study. 
Authors are grateful to all the study participants.

\section{REFERENCES}

[1] Begum M, Akter P, Hossain MM, et al. A comparative study between efficacy of esmolol and lignocaine for attenuating haemodynamics response due to laryngoscopy and endotracheal intubation. Faridpur Med Coll J 2010;5(1):25-8.

[2] Edwards ND, Alford AM, Dobson PM, et al. Myocardial ischemia during tracheal intubation and extubation. $\mathrm{Br} J$ Anaesth 1994;73(4):537-9.

[3] Sheppard S, Eagle CJ, Strunin L. A bolus dose of esmolol attenuate tachycardia and hypertension after tracheal intubation. Can J Anaesth 1990;37(2):202-5.

[4] Shepard LC, Gelman S, Reeves JG. Humoral response of hypertensive patients to laryngoscopy. Anesth Analg 1981;60:276-7.

[5] Coleman AJ, Jordan C. Cardiovascular response to anaesthesia. Influence of beta-adrenoreceptor blockade with metoprolol. Anaesthesia 1980;35(10):972-8.

[6] Devault M, Griefenstein FE, Harris IC. Circulatory response to endotracheal intubation in light general anaesthesia, the effect of atropine and phentolamine. Anaesthesiology 1960;21:360-2.

[7] Bhandari G, Shahi KS. Effect of gabapentin on pressor response to laryngoscopy and tracheal intubation: a double blind randomized placebo controlled study. People's J Sci Res 2013;6(1):1-6.

[8] Bhandari G, Mitra S, Shahi KN, et al. Pre-emptive use of oral pregabalin attenuates the pressor response of laryngoscopy and endotracheal intubation: a double blind randomized placebo controlled study. Ann Int Med Den Res 2016;2(3):110-4.

[9] Grover VK, Sharma S, Mahajan RP, et al. Intranasal nitroglycerine attenuate pressor response to tracheal intubation in beta-blocker treated hypertensive patients. Anaesthesia 1987;42(8):884-7.

[10] Charuluxananan S, Kyokong O, Somboonviboon W, et al. Nicardipine versus lidocaine for attenuating the cardiovascular response to endotracheal intubation. J Anesth 2000;14(2):77-81.

[11] Menda F, Koner O, Sayin M, et al. Dexmedetomidine as an adjunct to anesthetic induction to attenuate haemodynamic response to endotracheal intubation in patients undergoing fast-track CABG. Ann Card Anaesth 2010;13(1):16-21.

[12] Gunes Y, Gunduz M, Ozcengiz D, et al. Dexmedetomidine remifentanil or propofol-remifentanil anaesthesia in patients undergoing intracranial surgery. Neurosurg Q 2005;15:122-6.

[13] Agarwal P, Bhalla S, Singh I. To study the efficacy of intravenous esmolol, lidocaine and diltiazem in attenuating haemodynamic response to laryngoscopy and intubation. Internet J Anaesthesiol 2010;28(1):1-7.

[14] Sintetos AL, Hulse J, Pritchett EL. Pharmacokinetics and pharmacodynamics of esmolol administered as an intravenous bolus. Clin Pharmacol Ther 1987;41(1):1127.

[15] Mikawa K, Ikegaki J, Maekawa N, et al. The effect of diltiazem on the cardiovascular response to tracheal intubation. Anaesthesia 1990;45(4):289-93.

[16] Fujii Y, Tanaka H, Saitoh Y, et al. Effects of calcium channel blockers on circulatory response to tracheal intubation in hypertensive patients: nicardipine versus Diltiazem. Can J Anaesth 1995;42(9):785-8.

[17] Mikawa K, Nishina K, Maekawa N, et al. Comparison of nicardipine, diltiazem and verapamil for controlling the cardiovascular responses to tracheal intubation. $\mathrm{Br} \mathrm{J}$ Anaesth 1996;76(2):221-6.

[18] Kumar S, Mishra MN, Mishra LS, et al. Comparative study of the efficacy of i.v. esmolol, diltiazem and magnesium sulphate in attenuating haemodynamic response to laryngoscopy and tracheal intubation. Indian J Anaesth 2003;47(1):41-4.

[19] Mahesh M, Sri AS. Attenuation of cardiovascular responses to laryngoscopy and intubation-a comparative study between IV esmolol hydrochloride and lignocaine hydrochloride. Ann Int Med Den Res 2016;2(6):38-45.

[20] Menkhaus PG, Reves JG, Kissin I, et al. Cardiovascular effects of esmolol in anesthetized humans. Anesth Analg 1985;64(3):327-34.

[21] Singh S, Laing EF, Owiredu WKBA, et al. Comparison of esmolol and lidocaine for attenuation of cardiovascular stress response to laryngoscopy and endotracheal intubation in a Ghanaian population. Anesth Essays Res 2013;7(1):83-8

[22] Gupta C, Rastogi B, Agarwal S, et al. Clinical efficacy of esmolol, lignocaine, and diltiazem as premedicant for attenuation of haemodynamic responses of laryngoscopy and endotracheal intubation- a comparative evaluation. Int J Res Med Sci 2017;5(10):4443-9.

[23] Parvez G, Ommid M, Gupta AK, et al. Attenuation of the pressor response to laryngoscopy and tracheal intubation with intravenous diltiazem and esmolol intravenous in controlled hypertensive surgical patients. Rev Colomb Anestesiol 2010;38(4):457-69.

[24] Sarkar A, Kulkarni S, Thombre S. Attenuation of pressor response: a comparison of diltiazem and esmolol. Indian J Applied Res 2013;3(8):579-81.

[25] Stoelting RK. Circulatory changes during direct laryngoscopy and tracheal intubation: influence of duration of laryngoscopy with or without prior lidocaine. Anesthesiology 1977;47(4):381-4.

[26] Wang TL, Jiang Y, Yang BX. Effect of nicardipine combined with esmolol on systemic and tissue oxygenation during off-pump coronary artery bypass grafting surgery. Chin Med J 2005;118(2):130-5. 